\title{
Inactivation of AtRac1 by abscisic acid is essential for stomatal closure
}

\author{
Emmanuel Lemichez, ${ }^{1}$ Yan Wu, ${ }^{1}$ Juan-Pablo Sanchez, ${ }^{1}$ Amel Mettouchi, ${ }^{2}$ Jaideep Mathur, ${ }^{3}$ \\ and Nam-Hai Chua ${ }^{1,4}$ \\ ${ }^{1}$ Laboratory of Plant Molecular Biology, Rockefeller University, New York, New York 10021-6399, USA; ${ }^{2}$ Cellular \\ Biochemistry and Biophysics Program, Memorial Sloan-Kettering Cancer Center, New York, New York 10021, USA; \\ ${ }^{3}$ Laboratory of Plant Cell Biology, Institute of Molecular Agrobiology, National University of Singapore, Kent Ridge 117604, \\ Singapore
}

Plant water homeostasis is maintained by the phytohormone abscisic acid (ABA), which triggers stomatal pore closure in response to drought stress. We identified the Arabidopsis small guanosine triphosphatase (GTPase) protein AtRac1 as a central component in the ABA-mediated stomatal closure process. ABA treatment induced inactivation of AtRac GTPases and disruption of the guard cell actin cytoskeleton. In contrast, in the ABA-insensitive mutant abi1-1, which is impaired in stomatal closure, neither AtRac inactivation nor actin cytoskeleton disruption was observed on ABA treatment. These observations indicate that AtRac1 inactivation is a limiting step in the ABA-signaling cascade leading to stomatal closure. Consistent with these findings, expression of a dominant-positive mutant of AtRac1 blocked the ABA-mediated effects on actin cytoskeleton and stomatal closure in wild-type plants, whereas expression of a dominant-negative AtRac1 mutant recapitulated the ABA effects in the absence of the hormone. Moreover, the dominant-negative form of AtRac1 could also restore stomatal closure in abi1-1. These results define AtRac1 as a central element for plant adaptation to drought.

[Key Words: Abscisic acid; guard cell; actin; Rac; PP2C; turgor pressure]

Received April 2, 2001; revised version accepted May 29, 2001.

The closure of stomatal pores in aerial tissues is an important mechanism by which higher plants respond rapidly to water loss. This essential homeostatic response is regulated by the stress phytohormone ABA (Van Overbeek et al. 1967). ABA-induced stomatal closure requires different signaling components (Leung and Giraudat 1998; Leckie et al. 1998; Pei et al. 1998; Leyman et al. 1999; Li et al. 2000), one of which is ABI1, a member of the PP2C serine/threonine phosphatase family, which acts as an upstream regulator of the ABA pathway (Leung et al. 1994; Meyer et al. 1994; Gosti et al. 1999). The Arabidopsis abi1-1 mutant displays a wilty phenotype caused by impaired stomatal closure. Stomatal pores are bordered by a pair of guard cells. Under conditions of adequate water status, when the guard cells are fully turgid, constraints on their inner walls maintain the pore open. Closure of stomata has been ascribed to a release of the guard cell turgor pressure primarily caused by $\mathrm{Cl}^{-}$ and $\mathrm{K}^{+}$efflux (Blatt 2000). Additionally, a reorganization of the actin cytoskeleton of guard cells has been observed after ABA treatment (Eun and Lee 1997). Despite this observation, the precise role of the actin cytoskeleton in

\footnotetext{
${ }^{4}$ Corresponding author.

E-MAIL chua@rockvax.rockefeller.edu; FAX (212) 327-8327.

Article and publication are at http://www.genesdev.org/cgi/doi/10.1101/ gad.900401.
}

regulating stomatal relaxation has remained elusive because of the lack of characterized regulators of the plant actin cytoskeleton (Blatt 2000).

The family of Rho GTPases has emerged as a key regulator of the actin cytoskeleton in yeast and animal cells (Hall 1998). Through their interaction with multiple regulators and effectors, Rho GTPases in these organisms transduce signals from cell surface receptors to reorganize actin architecture in the cytoplasm. Small GTPases exist either in an active form bound to GTP or in an inactive form bound to GDP. The transition between the two forms is regulated by either GTPase-activating proteins (GAP) or GTP/GDP exchange factors (GEF), which inactivate or activate the GTPases, respectively (Bishop and Hall 2000). The association of GTPbound Rho GTPases with plasma membranes is stabilized by a carboxy-terminal prenyl extension. In contrast, soluble Rho GDP is maintained in the cytoplasm through its interaction with a GDP dissociation inhibitor (GDI).

The Arabidopsis database contains at least 13 distinct putative genes coding for Rho-related GTPases, some of which have been cloned (Xia et al. 1996; Winge et al. 1997; Li et al. 1998). Rho-related plant proteins have been described to regulate a complex array of biological processes, such as the regulation of pollen tube growth and plant cell death (Lin and Yang 1997; Kost et al. 
1999a,b; Kawasaki et al. 1999). Our study here defines a new involvement of Rho-related plant proteins in the control of stomatal closure and therefore plant water homeostasis.

\section{Results}

Arabidopsis AtRac1 is a plant Rho GTPase homolog

We have cloned a Rho-related Arabidopsis GTPase by screening for induction of abnormal cell shape after overexpression of Arabidopsis cDNAs in Schizosaccharomyces pombe (Xia et al. 1996). AtRacl is identical to ARAC3 (Winge et al. 1997) and Rop6At (Li et al. 1998). AtRac1 shares $60.9 \%, 47.7 \%$, and 51.85 homology with human Rac1, RhoA, and Cdc42, respectively, and is thus more related to mammalian Rac proteins than to RhoA and Cdc42. Rho-related GTPases bind to and hydrolyze GTP. The key amino acids involved in GTP binding and hydrolysis in mammalian small GTPases are conserved in AtRac1 (Fig. 1A). In particular, the three major residues that coordinate magnesium in the GTP bound form of Ras (T17, T35, and D57), as well as those involved in the binding of phosphate important for the intrinsic GTPase activity (G12, A59, and Q61), are strictly conserved (Pai et al. 1989). Based on the conservation of key

\section{A}
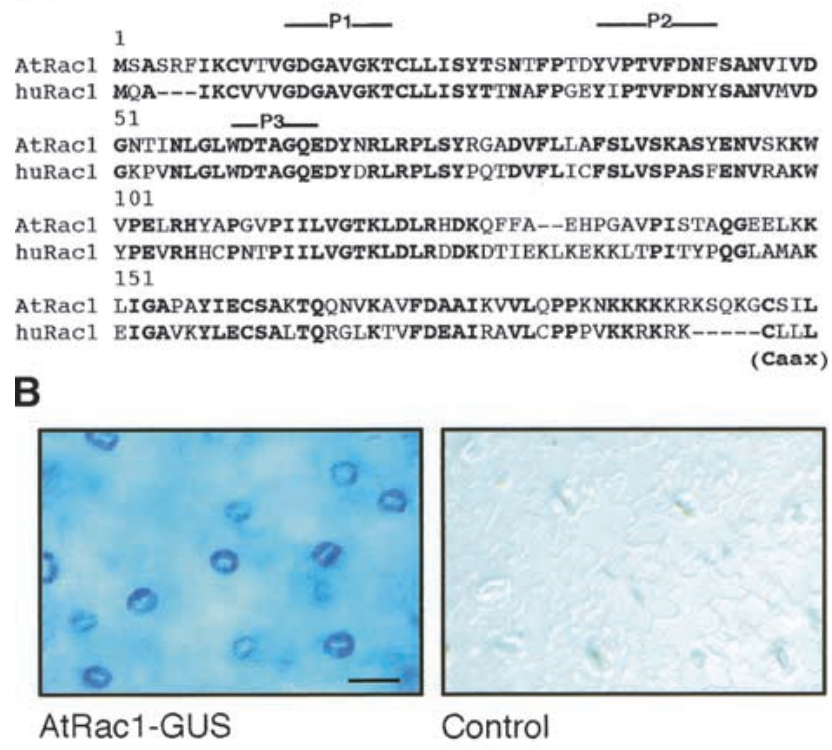

Figure 1. Amino acid sequence of AtRacl and AtRac1 expression in guard cells. (A) AtRacl amino acid sequence alignment with its close homolog human Racl Rho GTPase (huRac1). Conserved amino acids are shown in bold. The guanine nucleotide-binding domains are indicated by P1 to P3. The conserved prenylation motif, Caax, is noted. GenBank accession nos.: AtRac1 (U62746) and huRac1 (M29870). (B) GUS expression pattern in leaves of Arabidopsis WT (right) and AtRAC1 promoterGUS (left) transgenic seedlings. Three independent transgenic lines were analyzed, and all showed elevated GUS staining in guard cells over the epithelial background. Bar, $50 \mu \mathrm{m}$. amino acids, we predict that AtRacl should have similar biochemical characteristics to its mammalian Rho homologs. AtRac1 is ubiquitously expressed, having a stronger expression in vascular tissues (Kost et al. 1999a). Using AtRAC1 promoter $\beta$-glucuronidase (GUS) transgenic Arabidopsis plants (Kost et al. 1999a), we found that AtRacl is expressed at a much higher level in guard cells than in the surrounding epithelial cells (Fig. 1B). This observation and the known role of mammalian Rho GTPases in regulating the actin cytoskeleton led us to investigate whether the Arabidopsis small GTPase AtRacl is involved in the regulation of the actin cytoskeleton in guard cells.

\section{ABA-induced actin cytoskeleton disruption is impaired in abi1-1 plants}

Until recently, the extreme sensitivity of guard cells to biochemical treatments has seriously hampered studies of the actin cytoskeleton. Fixation techniques artificially release the guard cell turgor pressure and thus cause stomatal closure. The diffuse arrangement of actin that results has permitted only a correlation of actin reorganization with ABA treatment but not with stomatal closure per se (Eun and Lee 1997). To circumvent this technical difficulty and to simultaneously visualize both the state of the stomatal aperture and the organization of the actin cytoskeleton, we made use of transgenic Arabidopsis lines expressing a green fluorescent fusion protein (GFP-mTn) targeted to the actin cytoskeleton (Kost et al. 1998). We have previously generated two transgenic plant lines, WT/GFP-mTn and WT/MAP4-GFP, expressing GFP fusion proteins targeted to the actin (Kost et al. 1998) and tubulin (Mathur and Chua 2000) cytoskeletons, respectively. In WT/GFP-mTn guard cells of open stomata, we observed thick radial actin cables bridging the cell wall aperture to the dorsal side of the guard cell and a few tangential cables (Fig. 2). The microtubule cables of WT/MAP4-GFP guard cells were organized in a more regular fan-shaped pattern compared with that of actin (Fig. 2). On ABA addition, unlike microtubule cables, actin cables became rapidly disorganized and shortened. After $15 \mathrm{~min}$ of ABA treatment, $88 \%$ of the guard cells were closed and contained substantially fewer actin cables (Fig. 2). The guard cell actin cytoskeleton is thus specifically disorganized on ABA treatment.

To further study the link between actin reorganization and ABA-induced stomatal closure, we analyzed the response of the actin cytoskeleton in the mutant abi1-1, which is impaired in ABA-induced stomatal closure. We generated abi1-1/GFP-mTn transgenic plants by crossing WT/GFP-mTn with the abi1-1 mutant. T3 abi1-1/ GFPmTn homozygous plants were analyzed for the effects of ABA on the actin cytoskeleton. We observed that during $\mathrm{ABA}$ treatment, both stomatal aperture and the actin cytoskeleton remained unaffected (Fig. 2). Together, our observations indicate that ABA induced a disorganization of actin cables in guard cells during stoma- 
Figure 2. Actin and tubulin cytoskeleton reorganization in Arabidopsis guard cells on abscisic acid (ABA)-induced stomatal closure. Transgenic Arabidopsis WT and abi1-1 plants expressing GFP-mTn (Kost et al. 1998) or MAP4-GFP (Mathur and Chua 2000) were analyzed for cytoskeleton reorganization before (-ABA) and after (+ABA) $15 \mathrm{~min}$ of treatment with $50 \mu \mathrm{M}$ ABA. Using confocal microscopy, actin was visualized with GFP-mTn, whereas tubulin was visualized with MAP4-GFP. Pictures represent the projection of serial confocal optical sections and are representative of at least 100 guard cells analyzed for each condition. Bar, $10 \mu \mathrm{m}$.

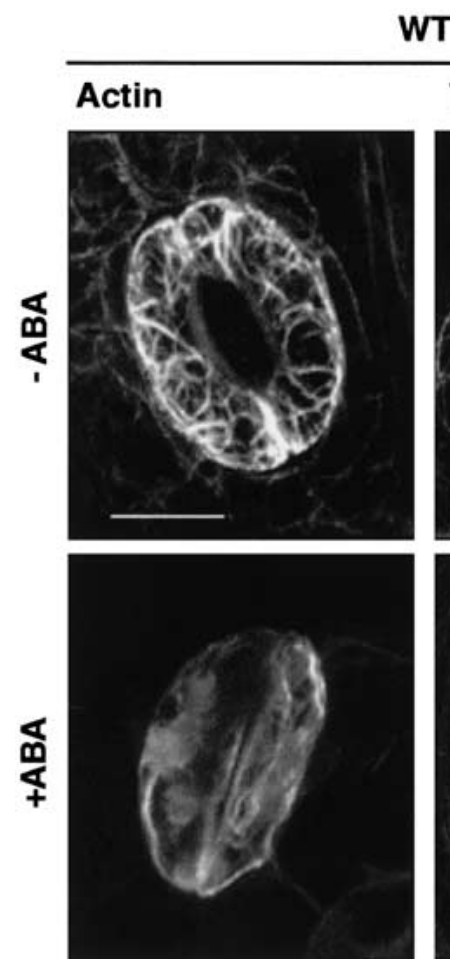

WT

\section{Tubulin}
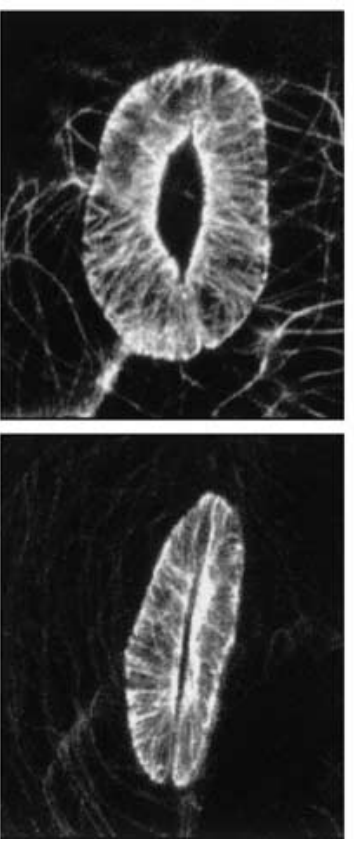

abi1
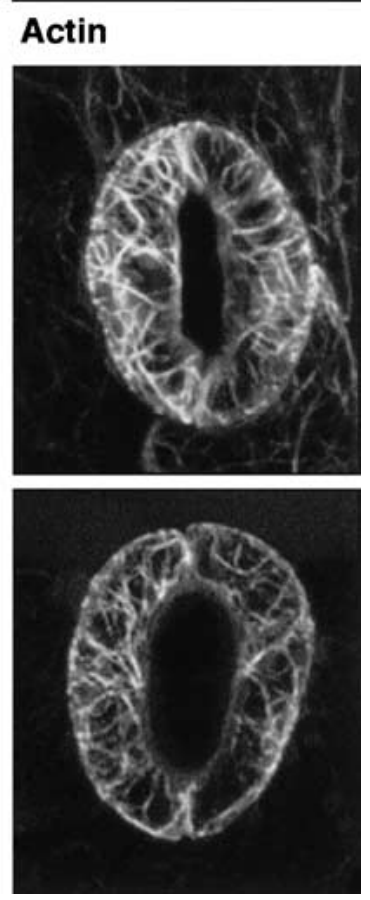

tal closure, a process impaired in the ABA-signaling mutant abi1-1. These results confirm and extend previous reports (Eun and Lee 1997; Eun et al. 2001).

\section{Conditional expression of AtRac1 mutants in transgenic Arabidopsis plants}

To further investigate the role of AtRacl in regulating the guard cell actin cytoskeleton, we generated dominant-negative (AtRac1-T20N) and dominant-positive (AtRac1-G15V) mutants, corresponding to the mammalian Rac-T17N (Ridley et al. 1992) and Rac-G12V (Diekmann et al. 1991), respectively. These types of mutants have been successfully used to uncover their specific effects on actin regulation in other systems (Hall 1998). WT and mutant AtRacls were purified as glutathione S-transferase (GST) fusion proteins in Escherichia coli (Self and Hall 1995a) and released from GST (Fig. 3A). WT AtRacl and mutant proteins were further characterized biochemically. AtRacl has a low intrinsic GTPase activity characteristic of small GTPases (Self and Hall 1995a). The half time of GTP hydrolysis was $17 \mathrm{~min}$, giving a rate constant $(\mathrm{k})$ of $0.04 \mathrm{~min}^{-1}$, which is more similar to that of RhoA ( $\left.k=0.039 \mathrm{~min}^{-1}\right)$ than to that of Rac1 ( $\mathrm{k}=0.069 \mathrm{~min}^{-1}$; Self and Hall 1995b). As expected, AtRac1-G15V displayed a 10-fold reduction in its intrinsic GTPase activity (Fig. 3B), whereas AtRac1-T20N showed a dramatic loss of its affinity for guanine nucleotides (Fig. 3C). Both mutants were tagged with the mycepitope so they could be differentiated from the endogenous AtRac on immunoblots. Transgenic Arabidopsis plants harboring the myc-tagged AtRacl mutants, under the control of a dexamethasone (dex)-inducible promoter (Aoyama and Chua 1997), were generated. Expression of both AtRacl mutant proteins was followed by immunoblotting. Figure 3D shows the typical sustained expression of AtRacl mutants during the first $48 \mathrm{~h}$ of dex treatment for two independent lines.

\section{Effect of AtRac1 mutants on guard cell actin cytoskeleton and stomatal closure}

Given their sequence homology with mammalian Rho family GTPases, it seems feasible that Arabidopsis Rho homologs might regulate the plant cell actin cytoskeleton. To address this question, we analyzed the effect of AtRac1 mutant expression on the guard cell actin cytoskeleton. WT/AtRac1 mutant transgenic lines were crossed with WT/GFP-mTn transgenic lines to generate AtRac1-T20N/GFP-mTn and AtRac1-G15V/GFP-mTn transgenic lines. T2 transgenic plants were verified for mutant AtRac1 induction and the GFP-mTn expression level (data not shown). On $48 \mathrm{~h}$ of dex-induction in continuous white light (WL) without ABA treatment, we observed a breakdown of actin cables in the majority of guard cells of AtRac1-T20N/GFP-mTn transgenic plants compared with those of the control. In contrast, we could not detect any significant changes in the guard cell actin cytoskeleton of AtRac1-G15V/GFP-mTn transgenic lines induced with dex (Fig. 4A). AtRac1-T20N expression can thus induce a breakdown of actin cables in guard cells, in a manner resembling the effects of ABA on the actin cables of WT guard cells.

We also found that expression of AtRac1-T20N could 

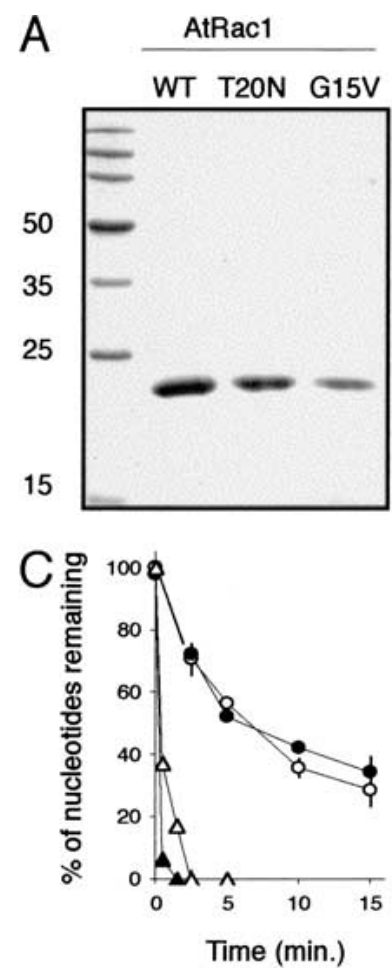

B
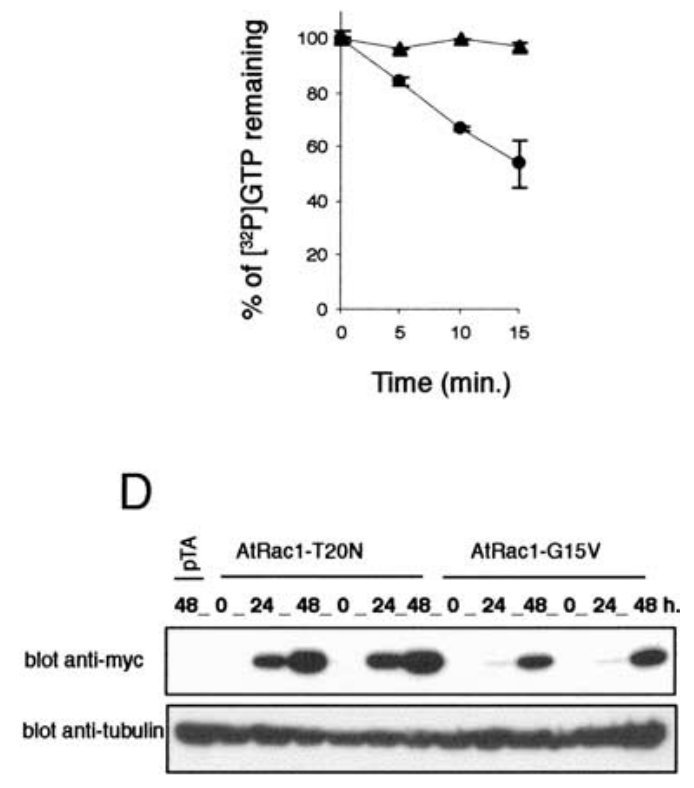

Figure 3. Biochemical characterization of AtRac1, and mutants and transgenic Arabidopsis plants showing inducible expression of AtRac1-mutants. (A) Coomassie blue staining of purified WT AtRacl (lane 1), AtRac1-T20N (lane 2), and AtRac1G15V (lane 3). Molecular mass markers are indicated on the left $(\mathrm{kD}) .(B)$ Kinetics of $\left[\gamma^{-}{ }^{32} \mathrm{P}\right] \mathrm{GTP}$ hydrolysis by WT AtRac1 (solid circles) and AtRac1-G15V (solid triangles). (C) Measurements of the $\left[{ }^{3} \mathrm{H}\right] \mathrm{GTP}$ released by WT AtRac1 (solid circles) and AtRac1-T20N (solid triangles) and $\left[{ }^{3} \mathrm{H}\right] \mathrm{GDP}$ released by WT AtRacl (hollow circles) and AtRac1-T20N (hollow triangles). (D) Anti-myc immunoblot showing the kinetics of dexamethasone-induced expression of AtRacl-mutants in two independent Arabidopsis transgenic lines for each mutant. pTA corresponds to transgenic plants carrying the empty vector. The amount of tubulin in the sample, as visualized by anti-tubulin antibodies, was used as a loading control.

induce stomatal closure, as observed on ABA-treatment of WT plants (Fig. 4A). To further analyze this observation, we measured the effect of AtRac1 mutant expression on stomatal aperture (Fig. 4B). WT/AtRac1-T20N plants were induced with dex for $48 \mathrm{~h}$ under WL. One leaf of each seedling was used to measure stomatal apertures, whereas the remainder of the seedling was processed for quantitation of AtRac1 mutant expression levels by Western blotting. We first verified that Arabidopsis transgenic lines containing the empty vector (pTA7002) remained sensitive to ABA, even after dex induction. Using WT/AtRac1-T20N transgenic plants, we observed that expression of the AtRacl dominantnegative mutant induced a closure of stomata, which is correlated with the expression level of AtRac1-T20N (Fig. 4B). In contrast, no stomatal closure was seen in either vector control or AtRac1-G15V plants after dex treatment (data not shown).

We next examined whether the constitutively active mutant of AtRac1 would display opposite effects on stomatal apertures. To this end, we compared the efficiency of ABA in inducing stomatal closure in dex-induced or noninduced WT/AtRac1-G15V transgenic plants under WL conditions. We observed that noninduced WT/ AtRac1-G15V plants were as sensitive as vector control transgenic plants to ABA with respect to stomatal closure. In contrast, expression of AtRac1-G15V interfered with the normal ABA-induced stomatal closure. Furthermore, this blocking effect by AtRac1-G15V was stronger in transgenic plants with a higher expression level of the transgene (Fig. 4B). In conclusion, we found that AtRac1 mutants have opposing effects on stomatal dynamics. Expression of a dominant-negative AtRac1 mutant mim- icked the ABA-induced actin disruption and stomatal closure, whereas a constitutively active AtRac1 mutant blocked the ABA-induced stomatal closure. In both cases, the physiological effect was related to the expression level of the AtRac1 mutant.

\section{$A B A$ induces AtRac inactivation in WT but not in abil-1 cells}

Because AtRac1-T20N recapitulates the ABA effects on actin cytoskeleton and stomatal closure, we investigated whether ABA acts by inactivating endogenous AtRac. We took advantage of the finding that only activated Rac can bind to the p- 21 activated kinase (PAK). This feature was used to pull down active Rac using GST fused to PAK (residues 70 to 106; Manser et al. 1998). Using transgenic plants expressing AtRacl mutants, we determined that AtRac1-G15V bound 62.8-fold more to GST-PAK ${ }^{70-106}$ than AtRac1-T20N, showing the specificity of this assay in plants (Fig. 5A). Because the binding of GTP-loaded AtRac1 to GST-PAK ${ }^{70-106}$ was 100fold lower than that of mammalian Rac1, this biochemical assay required the use of higher protein amounts (Fig. 5B). To overcome this technical difficulty, we generated cell cultures from Arabidopsis WT and abi1-1 mutant. ABA sensitivity of the cell lines was verified by following the induction of the early ABA responsive gene KIN2 (Kurkela and Borg-Franck 1992). KIN2 transcript was detected in WT cells 30 min after ABA addition, whereas no KIN2 transcript was detected in similarly treated abi1-1 cells (Fig. 5C). Having established the specificity of AtRacl binding to GST-PAK ${ }^{70-106}$ and the sensitivity 

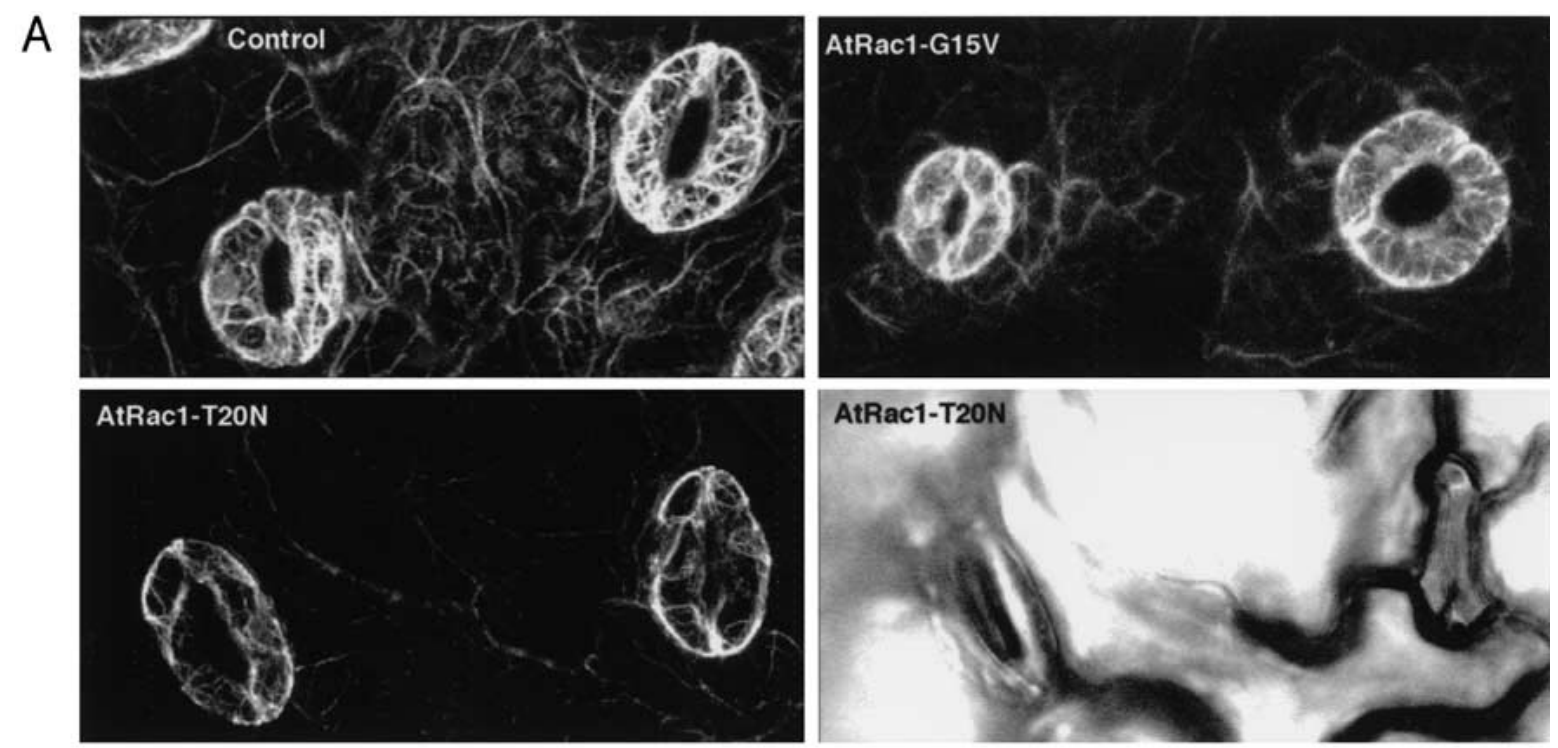

B

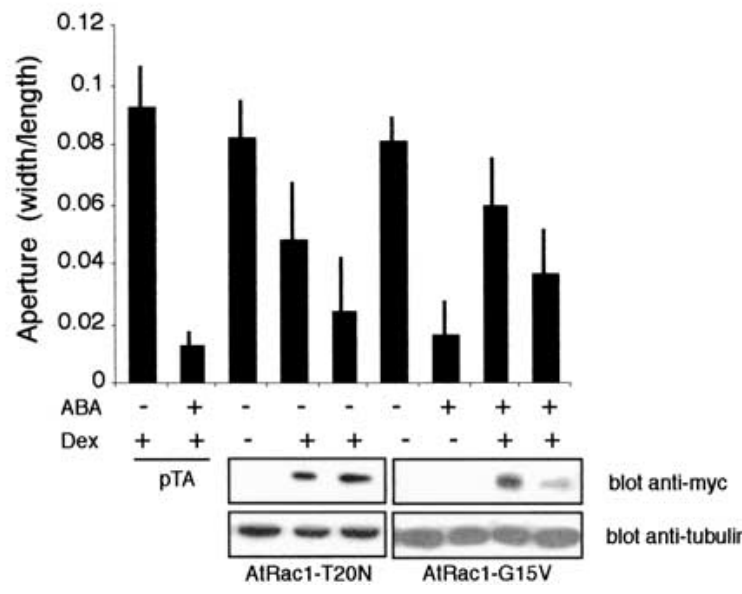

Figure 4. Effects of AtRac1-mutant expression on guard cells (A) Two-week-old transgenic seedlings of $p T A / G F P-m T n$ (control), AtRac1-T20N/GFP-mTn (dominant-negative mutant) and AtRac1-G15V/GFP-mTn (dominant-positive mutant) were induced for $24 \mathrm{~h}$ with $10 \mu \mathrm{M}$ dexamethasone (dex) in continuous white light (WL). The actin cytoskeleton was visualized with GFP-mTn using confocal microscopy. Pictures represent the projection of serial confocal optical sections. Note that AtRac1-T20N expression promotes actin cable disruption (lower left panel) and stomatal closure, as observed by light microscopy (lower right panel). For each condition, at least 50 guard cells were analyzed, and transgene expression was verified (not shown). (B) Measurement of stomatal aperture on transgenic seedlings induced for AtRac1 mutant expression. Expression of AtRac1-T20N induced stomatal closure, whereas AtRac1-G15V blocked the abscisic acid (ABA)induced stomatal closure. Both effects occurred in a dosedependent manner, as shown on immunoblots. Seedlings were incubated with $10 \mu \mathrm{M}$ dex for $48 \mathrm{~h}$ in WL conditions, followed by a 1-h ABA(10 $\mu \mathrm{M})$-treatment when indicated. One leaf of each seedling was used to measure the stomatal aperture. The graph represents the mean width of 60 stomata. The effects plotted in the graph were verified in three independent experiments. Error bars show the standard deviations. The remaining tissues were used to quantify transgene expression using Western blotting (lower panels). The anti-myc immunoblot shows the level of dex-induced expression of AtRac1 mutants, whereas the anti-tubulin immunoblot was used as a loading control. Measurements were performed on different trangenic lines and plotted on the graph to show that the effects on stomatal aperture are related to the expression levels of AtRacl mutants.

of Arabidopsis cells to ABA, we tested the effects of ABA on AtRac biochemical activity. We observed that addition of ABA to WT Arabidopsis cells inactivated AtRac in a time-dependent manner (Fig. 5D), with a half time of 15 min (Fig. 5F). This ABA effect on AtRac inactivation was dose dependent (Fig. 5E). In contrast to WT cells, we never observed AtRac inactivation in abi1-1 cells, which are insensitive to ABA (Fig. 5F,G). These results established that AtRac is specifically inactivated in Arabidopsis WT cell lines on ABA-treatment and indicate that AtRacl is likely inactivated on ABA-treatment of plants. Together with the observation that AtRacl-dominant mutants interfere with the stomatal closure (Fig. 4B), our results indicate that AtRacl inactivation could represent a limiting step in ABA-induced stomatal closure.
Expression of AtRac1 dominant-negative mutant can rescue stomatal closure in abil-1

To investigate whether AtRacl inactivation was required to allow the ABA-induced stomatal closure, we made use of our previous observations that $\mathrm{ABA}$ induces neither actin cable disruption nor AtRac inactivation in the abi1-1 mutant. To test whether AtRacl inactivation limits ABA-induced stomatal closure in abil-1 we crossed abi1-1 plants with inducible AtRac1 mutant transgenic plants. T2 lines were screened for abi1-1 homozygosity using PCR amplification (Fig. 6A). T3 abi11/AtRac1 mutant transgenic lines were tested for AtRac1 effects on stomatal aperture (Fig. 6B). We first verified that in the absence of the inducer abi1-1/At- 


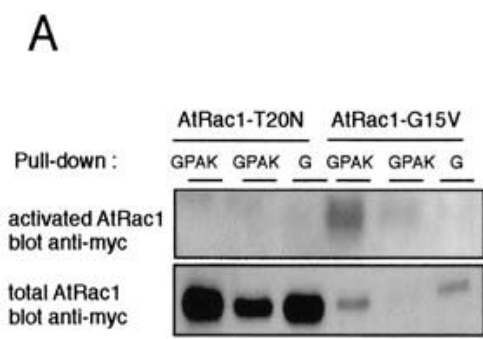

C

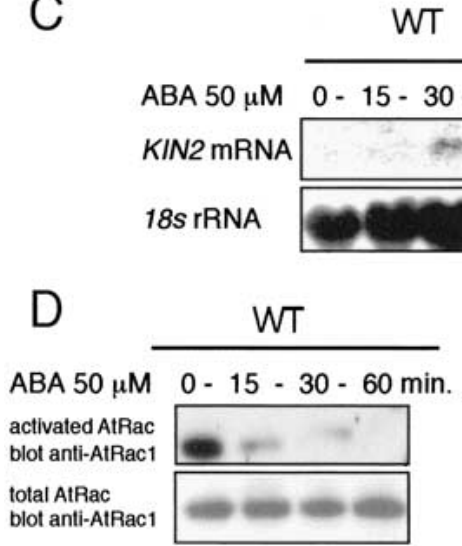

$a b i-1$

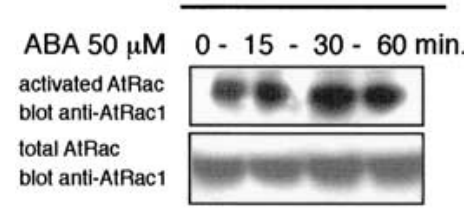

$\mathrm{F}$
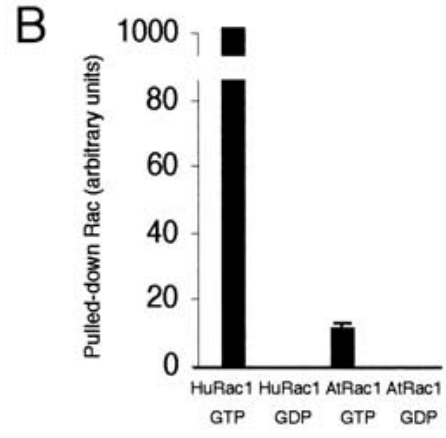

$a b i-1$

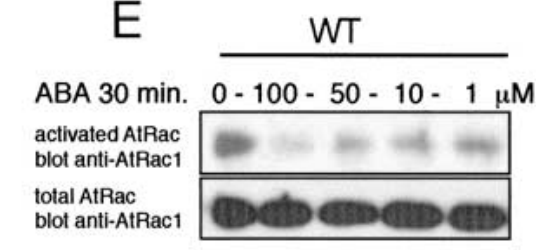

G

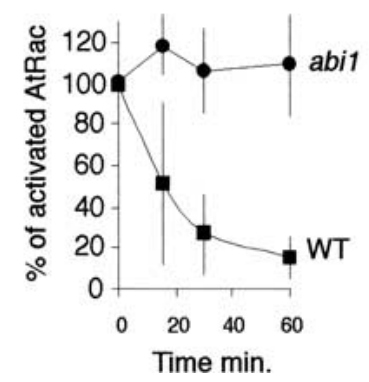

Figure 5. Abscisic acid (ABA) induces inactivation of AtRac in WT but not in abi1-1 cells. (A) Activated AtRac1 (AtRac1-G15V) binds specifically GST-PAK ${ }^{70-106}$. WT/ AtRacl-mutants transgenic plants were dexamethasone-induced for $48 \mathrm{~h}$ and processed for the GST-PAK ${ }^{70-106}$ pull-down assay. (Upper panel) Anti-myc immunoblots showed that AtRac1-G15V (active) but not AtRac1-T20N (inactive) bound to GSTPAK $^{70-106}$ (GPAK) and not to GST (G). (Lower panel) $10 \mu \mathrm{L}$ of each lysate was used to compare the amount of AtRac1-mutant engaged for each pull down. (B) In vitro affinity comparison of HuRacl and AtRacl binding to GST PAK ${ }^{70-106}$. Recombinant activated AtRacl (GTP-loaded) binds specifically to GST-PAK ${ }^{70-106}$, with a 100-fold lower affinity than HuRacl. (C) ABA-induced KIN2 transcript levels in Arabidopsis WT and abil-1 suspension cells. (Lower panel) 18S rRNA loading control. $(D-G)$ Effects of ABA on AtRac biochemical activity, using ABA-sensitive WT and ABA-insensitive abi1-1 cells. (D) Kinetics of ABA-mediated inactivation of AtRac1. Cells were treated with $50 \mu \mathrm{M}$ ABA and active AtRac1 determined by pull-down experiments. $(E)$ Dose response of the ABA-induced AtRac inactivation after $30 \mathrm{~min}$ of ABA-treatment. $(F)$ AtRacl is not inactivated by $\mathrm{ABA}$ in abi1-1 cells. $(G)$ Quantitation of the ABAinduced inactivation of AtRacl in WT and abi1-1 suspension cells measured by GST$\mathrm{PAK}^{70-106}$ pull down. Autoradiographs were quantified using N.I.H. Image 1.6 (average of four independent experiments with standard deviations).
Rac1-T20N plants remained insensitive to ABA. Using these plants, we observed that AtRac1-T20N expression induced stomatal closure, the extent of which was dependent on the expression level of the AtRacl dominant negative mutant.

\section{Discussion}

ABA-induced actin cable breakdown in guard cells is impaired in abil-1

Stomatal closure caused by guard cell relaxation is a dynamic process, allowing a plant to adapt rapidly to changes in environmental conditions. Recent studies have pointed to the importance of guard cell membrane dynamics during ABA-induced stomatal closure (Homann and Thiel 1999; Blatt 2000). Another related regulatory process of guard cell dynamics has emerged with the observation that the actin cytoskeleton might be disor- ganized during ABA-induced stomatal closure (Eun and Lee 1997). Notwithstanding this initial observation, the role of the actin cytoskeleton has remained elusive because of technical difficulties in imaging cytoskeletons in living guard cells. We have obviated this problem by using transgenic lines of Arabidopsis expressing either GFP-mTn (Kost et al. 1998) or MAP4-GFP (Mathur and Chua 2000), which allow noninvasive imaging of actin or tubulin cytoskeleton dynamics. We found that actin cables in guard cells were disrupted on ABA treatment. This breakdown was specific to the actin cytoskeleton because the microtubule cytoskeleton remained unchanged. Moreover, the actin cytoskeleton was unaffected by ABA treatment in the ABA-insensitive mutant abi1-1, which is impaired in stomatal closure. These observations, which confirm and extend earlier work (Eun and Lee 1997; Eun et al. 2001), reinforce the hypothesis that the actin cytoskeleton disruption is linked to stomatal closure. 
A

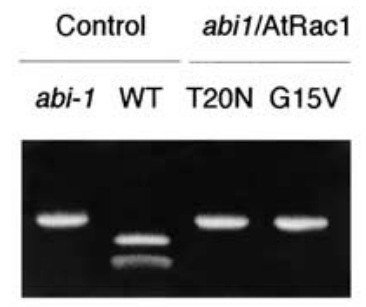

B

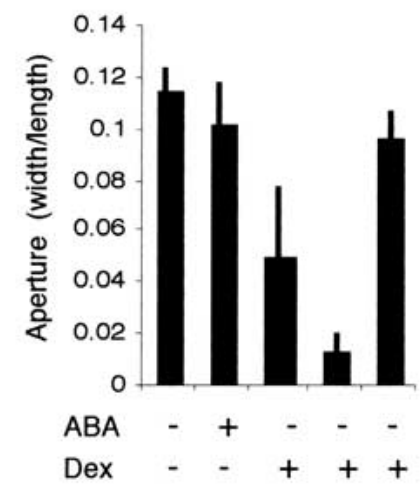

blot anti-myc

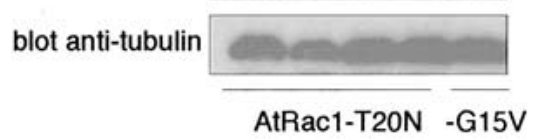

Figure 6. AtRac1-T20N expression can rescue stomatal closures in abi1-1. (A) Inducible AtRac1-mutant constructs were introduced into abi1-1 plants by crossing. The ethidium bromide DNA gel electrophoresis shows the endonuclease NcoI polymorphism on abi1-1 PCR products obtained from genomic DNAs of WT Arabidopsis ecotype Columbia, abi1-1 and the generated $\mathrm{F}_{2}$ plants abi1-1/AtRac1-mutants. $(B)$ Dexamethasone (dex)-induction of AtRac1-T20N but not AtRac1-G15V can rescue stomatal closure in abi1-1. Seedlings were incubated with $10 \mu \mathrm{M}$ dex for $48 \mathrm{~h}$ in continuous white light. One leaf of each seedling was used to measure the stomatal aperture. The graph represents the mean width value of 60 stomata with standard deviations. The effects plotted in the bar charts were verified in three independent experiments. The remaining tissues were used to quantify transgene expression using Western blotting (lower panels). Immunoblot anti-tubulin was used as a loading control. Note that AtRac1-T20N restores stomatal closure in abi1-1 in a dose-dependent manner.

\section{AtRac1 mutants interfere with $A B A$-induced actin cable disorganization and stomatal closure}

The finding that ABA treatment leads to actin cable disorganization raises the question of how the ABA signal is transduced and the identity of the components of the pathway for this process. In yeast and animal cells, Rho proteins are known to be upstream regulators of actin networks (Hall 1998). This finding, along with our observation that AtRacl is expressed in Arabidopsis guard cells, led us to investigate the possible roles of AtRacl in regulating both guard cell actin cytoskeleton dynamics and stomatal closure. One important feature of RhoGTPases is that dominant-positive and dominantnegative mutants can be generated to decipher their signaling roles (Bishop and Hall 2000). Dominant-negative mutants are thought to poison their exchange factors, thereby blocking activation of downstream effectors, whereas dominant-positive mutants remain insensitive to GTPase activating proteins, resulting in constitutive activation of their effectors.

Several lines of evidence presented in this paper support the notion that AtRacl is a key player in ABA-triggered guard cell actin disorganization and stomatal closure. First, expression of the dominant-negative mutant AtRac1-T20N can recapitulate the effects of ABA (Fig. 4). Second, In WT plants, the effects of ABA can be blocked by the dominant-positive mutant AtRac1-G15V (Fig. 4). Third, in the ABA-insensitive mutant abi1-1, AtRac1T20N, but not AtRac1-G15V, can induce stomatal closure (Fig. 6). In all three cases, the effect was correlated with the expression level of the appropriate AtRac1 mutant indicating that this small GTPase regulates a ratelimiting step.

\section{ABA treatment inactivates AtRac1}

Because AtRac1-T20N can recapitulate the effects of ABA on actin cable disorganization and stomatal closure, we hypothesize that one consequence of ABA signaling in guard cells is to inactivate AtRac1. Rho GTPases in other systems have been shown to be activated by many different growth factors, but almost nothing is known concerning external factors that may be responsible for their down-regulation. As it is technically difficult to obtain sufficient quantities of guard cells for biochemical analyses of AtRac1, we resorted to the use of Arabidopsis tissue culture cells. We first monitored expression of the ABA-responsive gene KIN2 in our WT and abi1-1 cell cultures to ensure that these cultures retain their appropriate ABA responses (Fig 5C). In addition, we modified a biochemical assay to measure the level of activated AtRac in Arabidopsis. In vitro, 1\% of AtRac1 GTP-loaded molecules bind to GST-PAK ${ }^{70-106}$. This low affinity binding probably reflects the two substitutions V/I33 and F/Y40 in the effector domain of AtRacl compared with that of the human Rac. This low interaction affinity can be overcome by using high protein concentrations of Arabidopsis cell lysates. Using this biochemical assay, we showed that indeed AtRac is inactivated on ABA treatment. The AtRac inactivation was dependent on the ABA concentration used and occurred with a half time of $15 \mathrm{~min}$. The time course of AtRac inactivation strongly correlated with the kinetics of stomatal closure induced by ABA. Moreover, ABA did not inactivate AtRac in abi1-1, which is insensitive to ABA (Fig. 5). Taken together, these results support the hypothesis that ABA induces stomatal closure through inactivation of AtRacl and that a block of AtRacl inactivation by ABA may be responsible for the impairment of stomatal closure in the abi1-1 mutant. Our results reveal a new pathway for the down-regulation of small 
Rho GTPases by ABA, which is dependent on the protein phosphatase IIC, ABI1. Given the emergence of guard cells as a popular system with which to dissect complex signaling mechanisms within a single cell, it will be of considerable interest to identify additional molecular components that govern stomatal opening and thus plant water status. Changes in guard cell volume require large adjustments in the surface area of the guard cell plasma membrane. Recent evidence indicates that extensive and rapid exocytic and endocytic events can account for the osmotically driven changes in guard cell surface area, which regulates stomatal aperture (Homann and Thiel 1999). Because the vesicles that add or remove membrane material must traffic along the actin cytoskeletal network, the possible involvement of ABAmediated inactivation of AtRacl in preventing the efficient shuttling between cytoplasmic vesicles and the plasma membrane requires further investigation.

\section{Materials and methods}

\section{Arabidopsis transgenic plants and suspension cell culture}

The AtRac1-GUS construct was previously described (Kost et al. 1999a). AtRac1-G15V and AtRac1-T20N mutants were generated by targeted mutagenesis on pAt043 encoding AtRac1 (Xia et al. 1996) and were tagged with sequences encoding myc by PCR amplification. cDNAs were subcloned into the XhoI-SpeI sites in the dex-inducible vector pTA7002 derived from pBI101/ 121 (Clontech) and into the EcoRI site of pGEX-2T (Pharmacia). WT Arabidopsis thaliana ecotype Columbia was transformed with pTA7002-AtRac1 mutants or the empty vector pTA7002 (pTA) by vacuum-infiltration (Bechtold et al. 1993). Transformants were selected on $100 \mu \mathrm{g} / \mathrm{mL}$ hygromycin containing MS medium supplemented with $3 \%$ sucrose and $0.8 \%$ agar. Plants were transferred onto MS medium containing $10 \mu \mathrm{M}$ dex (D4902, Sigma) to induce expression of AtRacl mutants. Transgenic plants carrying AtRac1 mutant and pTA vector control (Fig. 2C) were crossed with plants carrying the GFP-mTn construct (Kost et al. 1998) to generate GFP-mTn/AtRacl mutant or GFP-mTn/pTA transgenic plants. Transgenic abil/AtRac1 mutant plants were obtained by crossing WT/AtRac1 mutants with abi1-1. Transgenic abil/GFP-mTn plants were obtained by crossing WT/GFP-mTn with abi1-1. All transgenic abi1-1 homozygous plant lines were screened by PCR amplification using DNA from T2 and T3 lines as described previously (Pei et al. 1998). Cell suspension cultures from WT and abi1-1 A. thaliana were generated as previously described (Mathur and Koncz 1998). Cells were propagated at $22^{\circ} \mathrm{C}$ in darkness in MS medium supplemented with twofold $\mathrm{B}_{5}$-vitamins, $3 \%$ sucrose, $1 \mathrm{mg} / \mathrm{L}$ 2,4-dichlorophenoxyacetic acid, and $0.5 \mathrm{mg} / \mathrm{L}$ 6- $(\gamma, \gamma$-dimethylallylamino)-purine riboside.

\section{Histochemical and fluorescent microscopy analyses}

AtRac1-GUS transgenic seedlings were incubated overnight at $37^{\circ} \mathrm{C}$ with gentle rolling in $50 \mathrm{mM}$ Tris- $\mathrm{HCl}(\mathrm{pH} 7.0)$ containing $0.2 \%$ X-Gluc (Jersey Lab and Gloves Supply), 1\% Triton X-100, $5 \mathrm{mM}$ potassium ferricyanide, and $5 \mathrm{mM}$ potassium ferrocyanide. Seedlings were treated $48 \mathrm{~h}$ with $70 \%$ ethanol and mounted in $50 \%$ glycerol to prevent tissue dehydration. GUS staining was analyzed by bright-field transmitted light microscopy using an Axioscope (Carl Zeiss Inc.) microscope. Images were taken by $35-\mathrm{mm}$ photography (63t film; Eastman Kodak). Confocal analysis of GFP expression was performed using an
LSM410 inverted microscope (Carl Zeiss) as previously described (Kost et al. 1998). Transgenic GFP-mTn and GFP-MAP4 seedlings were submerged intact in $10 \mathrm{mM}$ MES-Tris (pH 6.1),

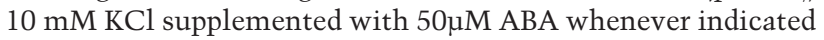
and observed.

\section{GTPase activity and nucleotide exchange assays}

Recombinant AtRac1 WT and mutants were affinity-purified from E. coli lysates as GST fusion proteins and released from GST using thrombin as previously described (Self and Hall 1995a). The intrinsic AtRac1 and AtRac1-G15V GTPase activity was measured using the nitrocellulose filter-binding method (Self and Hall 1995b). The intrinsic nucleotide exchange activity of AtRac1 and AtRac1-T20N was measured at high magnesium concentrations using the nitrocellulose filter-binding method (Self and Hall 1995b).

\section{Pull-down methods}

AtRac inactivation was assessed using the GST-PAK ${ }^{70-106}$ pulldown method (Manser et al. 1998). For the in vitro affinity comparison, AtRacl and huRac1 proteins were loaded with GTP or GDP (Self and Hall 1995b) and assessed for GST-PAK ${ }^{70-106}$ binding as previously described (Benard et al. 1999). To measure the in vivo AtRac biochemical activities, $1 \mathrm{~mL}$ of pelleted suspension cells or ten 2-week-old seedlings were ground in $1.5 \mathrm{~mL}$ of the IP buffer (40 mM HEPES at $\mathrm{pH} 7.5,100 \mathrm{mM} \mathrm{NaCl}, 5 \mathrm{mM}$ $\mathrm{MgCl}_{2}, 4 \%$ glycerol, $0.5 \%$ Triton X-100, $10 \mathrm{mM} \mathrm{NaF}, 20 \mathrm{mM}$ $\beta$-glycerophosphate supplemented freshly with $2 \mathrm{mM} \mathrm{Na-Vana-}$ date, $5 \mathrm{mM}$ DTT, $1 \mathrm{mM}$ PMSF, and protease inhibitors complete EDTA-free; Roche); $2.5 \mathrm{mg}$ of the clarified lysates was used in each pull-down assay. In parallel, $25 \mu \mathrm{g}$ of each lysate was used to determine the amount of total-AtRac by Western blotting. Western blots were performed after SDS-PAGE fractionation and transfer to immobilon-P membranes (Millipore) at a constant voltage $35 \mathrm{~V}$, using carbonate buffer. Anti-Myc monoclonal 9E10, anti- $\beta$-tubulin (Amersham Life Science), and immunopurified AtRac1 polyclonal antibodies were used at $1 \mu \mathrm{g} /$ $\mathrm{mL}$, followed by anti-mouse or anti-rabbit horseradish peroxi dase incubation (1:3000, Amersham Life Science). Immunoreactive bands were visualized using ECL-plus (Amersham Life Science). Polyclonal antiserum against GST-AtRac1 was immunopurified on recombinant AtRac1. Immunopurified anti-AtRacl used at $1 \mu \mathrm{g} / \mathrm{mL}$ enables detection of $5 \mathrm{fmoles}$ of purified AtRac1. AntiAtRacl could detect other AtRac members, referred to as AtRac when Western blots were performed on total cell extracts.

\section{Stomatal aperture measurements}

Measurements were performed on 2-week-old seedlings induced for $48 \mathrm{~h}$ with $10 \mu \mathrm{M}$ dex in WL conditions. Seedlings were incubated for $30 \mathrm{~min}$ in $10 \mathrm{mM}$ MES-Tris (pH 6.1), $10 \mathrm{mM} \mathrm{KCl}$ and supplemented with 10 to $50 \mu \mathrm{M}$ ABA $([ \pm]$-cis,trans-ABA; Sigma) when indicated. Widths and lengths of stomatal pores were measured using a LSM410 inverted confocal microscope (Carl Zeiss). Each value corresponds to the average of 60 stomata measured on one leaf, along with the corresponding standard deviation. The remainder of each seedling was processed for Western blot analysis of AtRac1 mutants to correlate the expression level with the effects on stomatal aperture. One representative experiment out of three was plotted for each graph.

\section{Northern blotting}

Total RNA was isolated from $1 \mathrm{~mL}$ of cell pellet using the QIAGEN purification kit. Each lane contained $10 \mu \mathrm{g}$ total RNA. Northern blot hybridization was performed as described previously (Ausubel et al. 1994). 


\section{Acknowledgments}

We thank Drs. L. Lim, P. Hare, K. Kirsch, S.G. Møller, Y. Sun, and L. Lopez-Molina, for materials, advice, and discussion. This work was supported by DOE grant DOE94ER20143 (to N.H.C.) and by a Human Frontier Science Program Post-doctoral fellowship LT 256/97 to E.L.

The publication costs of this article were defrayed in part by payment of page charges. This article must therefore be hereby marked "advertisement" in accordance with 18 USC section 1734 solely to indicate this fact.

\section{References}

Aoyama, T. and Chua, N.-H. 1997. A glucocorticoid-mediated transcriptional induction system in transgenic plants. Plant J. 11: 605-612.

Ausubel, F.M., Brent, R., Kinston, R.E., Moore, D.D., Seidman, J.G., Smith, J.A., and Struhl, K. 1994. Current Protocols in Molecular Biology. Wiley, New York, NY.

Bechtold, N., Ellis, J., and Peletier G. 1993. In planta Agrobacterium-mediated gene transfer by infiltration of adult Arabidopsis thaliana plants. C.R. Acad. Sci. Paris Life Sci. 316: 1194-1199.

Benard, V., Bohl, B.P., and Bokoch, G.M. 1999. Characterization of rac and cdc42 activation in chemoattractant-stimulated human neutrophils using a novel assay for active GTPases. J. Biol. Chem. 274: 13198-13204.

Bishop, A.L. and Hall, A. 2000. Rho GTPases and their effector proteins. Biochem. J. 348: 241-255.

Blatt, M.R. 2000. Cellular signaling and volume control in stomatal movements in plants. Annu. Rev. Cell Dev. Biol. 16: 221-241.

Diekmann, D., Brill, S., Garrett, M.D., Totty, N., Hsuan, J., Monfries, C., Hall, C., Lim, L., and Hall, A. 1991. Bcr encodes a GTPase-activating protein for p21rac. Nature 351: 400-402.

Eun, S.-O. and Lee, Y. 1997. Actin filaments of guard cells are reorganized in response to light and abscisic acid. Plant Physiol. 115: 1491-1498.

Eun, S.-O. Bae, S.-H., and Lee, Y. 2001. Cortical actin filaments in guard cells respond differently to abscisic acid in wildtype and abi1-1 mutant Arabidopsis. Planta 212: 466-469.

Gosti, F., Beaudoin, N., Serizet, C., Webb, A.A.R., Vartanian, N., and Giraudat, J. 1999. ABI1 protein phosphatase 2C is a negative regulator of abscisic acid signaling. Plant Cell 11: 1897-1910.

Hall, A. 1998. Rho GTPases and the actin cytoskeleton. Science 279: 509-514.

Homann, U. and Thiel, G. 1999. Unitary exocytotic and endocytotic events in guard-cell protoplasts during osmotically driven volume changes. FEBS Lett. 460: 495-499.

Kawasaki, T., Henmi, K., Ono, E., Hatakeyama, S., Iwano, M., Satoh, H., and Shimamoto, K. 1999. The small GTP-binding protein Rac is a regulator of cell death in plants. Proc. Natl. Acad. Sci. 96: 10922-10926.

Kost, B., Spielhofer, P., and Chua, N.-H. 1998. A GFP-mouse talin fusion protein labels plant actin filaments in vivo and visualizes the actin cytoskeleton in growing pollen tubes. Plant I. 16: 393-401.

Kost, B., Lemichez, E., Spielhofer, P., Hong, Y., Tolias, K., Carpenter, C., and Chua, N.-H. 1999a. Rac homologues and compartmentalized phosphatidylinositol 4,5-bisphosphate act in a common pathway to regulate polar pollen tube growth. J. Cell Biol. 145: 317-330.

Kost, B., Mathur, J., and Chua, N.-H. 1999b. Cytoskeleton in plant development. Cur. Opin. Plant Biol. 2: 462-470.

Kurkela, S. and Borg-Franck, M. 1992. Structure and expression of kin2, one of two cold- and ABA-induced genes of Arabidopsis thaliana. Plant Mol. Biol. 19: 689-692.

Leckie, C. P., McAinsh, M.R., Allen, G.J., Sanders, D., and Hetherington, A.M. 1998. Abscisic acid-induced stomatal closure mediated by cyclic ADP-ribose. Proc. Natl. Acad. Sci. 95: 15837-15842.

Leung, J. and Giraudat, J. 1998. Abscisic acid signal transduction. Annu. Rev. Plant Physiol. Plant Mol. Biol. 49: 199-222.

Leung, J., Bouvier-Durand, M., Morris, P.-C., Guerrier, D., Chefdor, F., and Giraudat, J. 1994. Arabidopsis ABA response gene ABIl: Features of a calcium-modulated protein phosphatase. Science 264: 1448-1452.

Leyman, B., Geelen, D., Quintero, F.J., and Blatt, M.R. 1999. A tobacco syntaxin with a role in hormonal control of guard cell ion channels. Science 283: 537-540.

Li, H., Wu, G., Ware, D., Davis, K.R., and Yang, Z. 1998. Arabidopsis Rho-related GTPases: Differential gene expression in pollen and polar localization in fission yeast. Plant Physiol. 118: 407-417.

Li, J., Wang, X.-Q., Watson, M.B., and Assmann, S.M. 2000. Regulation of abscisic acid-induced stomatal closure and anion channels by guard cell AAPK kinase. Science 287: 300-303.

Lin, Y. and Yang, Z. 1997. Inhibition of pollen tube elongation by microinjected anti-Rop1Ps antibodies suggests a crucial role for Rho-type GTPases in the control of tip growth. Plant Cell 9: 1647-1659.

Manser, E., Loo, T.-H., Koh, C.-G., Zhao, Z.-S., Chen, X.-Q., Tan, L., Tan, I., Leung, T., and Lim, L. 1998. PAK kinases are directly coupled to the PIX family of nucleotide exchange factors. Mol. Cell 1: 183-192.

Mathur, J. and Koncz, C. 1998. Protoplast isolation, culture, and regeneration. Methods Mol. Biol. 82: 35-42.

Mathur, J. and Chua, N.-H. 2000. Microtubule stabilization leads to growth reorientation in Arabidopsis trichomes. Plant Cell 12: 465-477.

Meyer, K., Leube, M.P., and Grill, E. 1994. A protein phosphatase 2C involved in ABA signal transduction in Arabidopsis thaliana. Science 264: 1452-1455.

Pai, E.F., Kabsch, W., Krengel, U., Holmes, K.C., John, J., and Wittinghofer, A. 1989. Structure of the guanine-nucleotidebinding domain on the ha-ras oncogene product $\mathrm{p} 21$ in the triphosphate conformation. Nature 341: 209-214.

Pei, Z.-M., Ghassemian, M., Kwak, C.M., McCourt, P., and Schroeder, J.I. 1998. Role of farnesyltransferase in ABA regulation of guard cell anion channels and plant water loss. Science 282: 287-290.

Ridley, A.J., Paterson, H.F., Johnston, C.L., Diekmann, D., and Hall, A. 1992. The small GTP-binding protein rac regulates growth factor-induced membrane ruffling. Cell 70: 401-410.

Self, A.J. and Hall, A. 1995a. Purification of recombinant Rho/ Rac/G25K from Escherichia coli. Methods Enzymol. 256: 310.

1995b. Measurement of intrinsic nucleotide exchange and GTP hydrolysis rates. Methods Enzymol. 256: 67-76.

Van Overbeek, J., Loeffler, J.E., and Mason, M.I. 1967. Dormin (Abscisin II), inhibitor of plant DNA synthesis? Science 156: 1497-1499.

Winge, P., Brembu, T., and Bones, A.M. 1997. Cloning and characterization of rac-like cDNAs from Arabidopsis thaliana. Plant Mol. Biol. 35: 483-495.

Xia, G., Ramachandran, S., Hong, Y., Chan, Y.S., Simanis, V., and Chua, N.-H. 1996. Identification of plant cytoskeletal, cell cycle-related and polarity-related proteins using Schizosaccharomyces pombe. Plant J. 10: 761-769. 


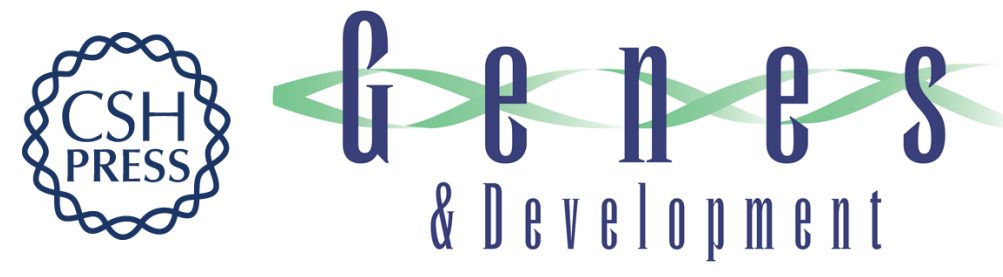

\section{Inactivation of AtRac1 by abscisic acid is essential for stomatal closure}

Emmanuel Lemichez, Yan Wu, Juan-Pablo Sanchez, et al.

Genes Dev. 2001, 15:

Access the most recent version at doi:10.1101/gad.900401

References This article cites 34 articles, 16 of which can be accessed free at: http://genesdev.cshlp.org/content/15/14/1808.full.html\#ref-list-1

License

Email Alerting Receive free email alerts when new articles cite this article - sign up in the box at the top Service right corner of the article or click here.

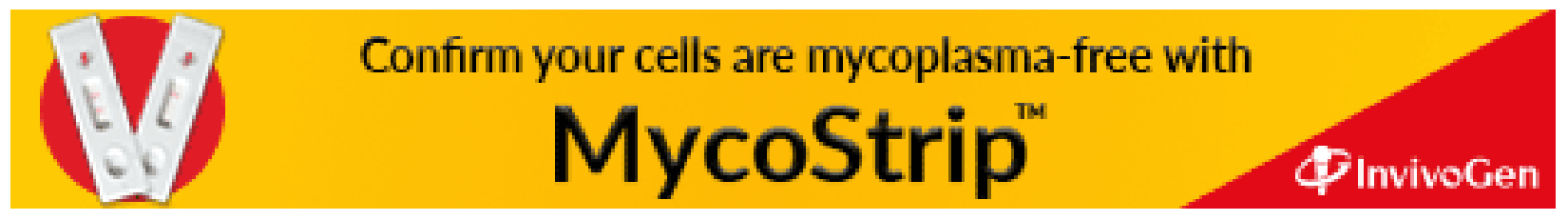

